\title{
Firing Rate of the Noisy Quadratic Integrate-and-Fire Neuron
}

\author{
Nicolas Brunel \\ brunel@biomedicale.univ-paris5.fr \\ CNRS, NPSM, Université Paris René Descartes, 75270 Paris Cedex 06, France
}

Peter E. Latham

pel@ucla.edu

Department of Neurobiology, University of California at Los Angeles, Los Angeles, CA 90095, U.S.A.

We calculate the firing rate of the quadratic integrate-and-fire neuron in response to a colored noise input current. Such an input current is a good approximation to the noise due to the random bombardment of spikes, with the correlation time of the noise corresponding to the decay time of the synapses. The key parameter that determines the firing rate is the ratio of the correlation time of the colored noise, $\tau_{s}$, to the neuronal time constant, $\tau_{m}$. We calculate the firing rate exactly in two limits: when the ratio, $\tau_{s} / \tau_{m}$, goes to zero (white noise) and when it goes to infinity. The correction to the short correlation time limit is $\mathcal{O}\left(\tau_{s} / \tau_{m}\right)$, which is qualitatively different from that of the leaky integrate-and-fire neuron, where the correction is $\mathcal{O}\left(\sqrt{\tau_{s} / \tau_{m}}\right)$. The difference is due to the different boundary conditions of the probability density function of the membrane potential of the neuron at firing threshold. The correction to the long correlation time limit is $\mathcal{O}\left(\tau_{m} / \tau_{s}\right)$. By combining the short and long correlation time limits, we derive an expression that provides a good approximation to the firing rate over the whole range of $\tau_{s} / \tau_{m}$ in the suprathreshold regimethat is, in a regime in which the average current is sufficient to make the cell fire. In the subthreshold regime, the expression breaks down somewhat when $\tau_{s}$ becomes large compared to $\tau_{m}$.

\section{Introduction}

A major challenge in computational neuroscience is to understand the behavior of large recurrent networks of spiking neurons. A first step in this endeavor is to be able to compute the location and stability of a network's equilibria, given connectivity and single neuron properties. For networks operating in the asynchronous regime, the equilibria can be determined by solving a set of algebraic equations in which the firing rate of each neuron in the network is a function of the firing rates of all the other neurons. To solve these equations, which can be done using mean-field techniques, it is 
necessary to be able to compute the mapping from presynaptic firing rates to postsynaptic rates. For leaky integrate-and-fire neurons, the mapping is known (Ricciardi, 1977; Tuckwell, 1988), and equilibria have been computed for networks of irregularly firing leaky integrate-and-fire neurons (Amit \& Brunel, 1997a, 1997b; Brunel, 2000). Here we compute this mapping for a more realistic reduced neuron model, the quadratic integrate-and-fire neuron (Ermentrout \& Kopell, 1986).

Cortical neurons in vivo fire in an irregular fashion (Burns \& Webb, 1976; Softky \& Koch, 1993), and intracellular recordings reveal large fluctuations in the membrane potential (Destexhe \& Paré, 1999; Anderson, Lampl, Gillespie, \& Ferster, 2000). These experimental observations suggest that the input to a neuron can be divided into two components, a mean current and fluctuations around that mean, both of which depend on the firing rates of the presynaptic neurons. It is often reasonable to approximate the fluctuations as colored noise with a correlation time proportional to the synaptic time constants (Amit \& Tsodyks, 1991; Brunel \& Sergi, 1998; Destexhe, Rudolph, Fellous, \& Sejnowski, 2001), and this is the approach we take here. In addition, we assume that there is a unique synaptic decay time, $\tau_{s}$, so that the correlation time of the noise is equal to $\tau_{s}$. With these assumptions, the input of the neuron is fully described by three parameters: the mean current, $\mu$; the variance of the fluctuations, $\sigma^{2}$; and the correlation time constant, $\tau_{s}$.

The firing rate of the leaky integrate-and-fire neuron versus $\mu$ and $\sigma$ was calculated by Brunel and Sergi (1998; see also Fourcaud \& Brunel, 2002), in the limit $\tau_{s} \ll \tau_{m}$ where $\tau_{m}$ is the membrane time constant. Although the leaky integrate-and-fire neuron is a popular model, it suffers from two drawbacks: it cannot be derived from conductance-based models using a rigorous reduction procedure, and its $f$-I curve in the absence of noise has a pathological behavior close to threshold. For these reasons, it is useful to consider another simplified model neuron: the quadratic integrate-andfire neuron. This neuron, which is related by a change of variables to the $\theta$-neuron (Ermentrout, 1996; Gutkin \& Ermentrout, 1998) represents the normal form of a neuron with a type I bifurcation leading to spike generation (Ermentrout \& Kopell, 1986; Ermentrout, 1996). The quadratic integrateand-fire neuron is thus expected to describe the dynamics of any type I neuron close to bifurcation, where firing rates are low. In particular, its firing rate in the absence of noise scales as $\sqrt{I-I_{t h r}}$ where $I_{t h r}$ is the threshold current. This is the behavior exhibited by all type I neurons near threshold.

Here we compute the firing rate of the quadratic integrate-and-fire neuron in two limits: small and large $\tau_{s} / \tau_{m}$. Interpolation between these limits provides a good approximation to the firing rate over the whole range of $\tau_{s} / \tau_{m}$ in the suprathreshold regime and a reasonable zeroth-order approximation in the subthreshold regime. The firing rate must be computed numerically, but for applications such as mean-field analysis, where speed is important, two lookup tables are sufficient to characterize the firing rate for all values of $\mu, \sigma, \tau_{m}$, and $\tau_{s}$. 


\section{Neuron Model and Analytical Methods}

The quadratic integrate-and-fire neuron with synaptic noise obeys the differential equations

$$
\begin{aligned}
\tau_{m} \frac{d v}{d t} & =f(v)+w \\
\tau_{s} \frac{d w}{d t} & =-w+\tau_{m}^{1 / 2} \sigma \eta(t)
\end{aligned}
$$

where $\tau_{m}$ is the neuronal time constant (hereafter called the membrane time constant), $v(t)$ is a "voltage" variable (hereafter called the membrane potential),

$$
f(v)=v^{2}+\mu
$$

with $\mu$ the mean input current flowing into the cell, $w$ represents the stochastic component in the synaptic currents, $\tau_{s}$ is the synaptic time constant, $\eta$ is white noise with $\left\langle\eta(t) \eta\left(t^{\prime}\right)\right\rangle=\delta\left(t-t^{\prime}\right)$, and $\sigma$ is the overall amplitude of the noise.

In the noiseless version of the model, when the membrane potential becomes positive, the quadratic term on the right-hand side of equation 2.1a ensures that the membrane potential diverges to infinity in finite time. The time at which the membrane potential reaches infinity defines the time a spike is emitted. Another way of defining the spike time would be to set a threshold for firing and then formally send this threshold to infinity. Immediately after firing, the membrane potential is reset to $-\infty$. Again, the quadratic term in the right-hand side of equation 2.1a ensures that the membrane potential comes back to a finite value in finite time. To determine the firing rate from equation 2.1, we reformulate the problem using a FokkerPlanck equation (Risken, 1984),

$$
\tau_{m} \partial_{t} P(v, w, t)+\partial_{v}[(f(v)+w) P]+\frac{\tau_{m}}{\tau_{s}} \partial_{w}[-w P]=\frac{\sigma^{2}}{2} \frac{\tau_{m}^{2}}{\tau_{s}^{2}} \partial_{w}^{2} P,
$$

where $P(v, w, t)$ is the joint probability density function (pdf) of $(v, w)$ at time $t$. Once $P(v, w, t)$ is known, the firing rate is given by the total probability flux at $v=+\infty$ (see equation 2.5 below). For other studies of the pdf approach to neural modeling, mostly in the context of integrate-and-fire neurons, see Treves (1993), Abbott and van Vreeswijk (1993), Brunel and Hakim (1999), Brunel (2000), Knight, Omurtag, and Sirovich (2000), and Nykamp and Tranchina (2000).

The Fokker-Planck equation can be rewritten as a continuity equation,

$$
\partial_{t} P(v, w, t)+\partial_{v} J_{v}(v, w, t)+\partial_{w} J_{w}(v, w, t)=0
$$


where $J_{v}$ and $J_{w}$ are probability fluxes,

$$
\begin{aligned}
& J_{v}(v, w, t)=\frac{1}{\tau_{m}}(f(v)+w) P(v, w, t) \\
& J_{w}(v, w, t)=\frac{1}{\tau_{s}}\left(-\frac{\tau_{m}}{\tau_{s}} \frac{\sigma^{2}}{2} \partial_{w} P(v, w, t)-w P(v, w, t)\right) .
\end{aligned}
$$

The firing rate, $v(t)$, is the total probability flux at $v=+\infty$; it is given by

$$
v(t)=\int_{-\infty}^{+\infty} d w \lim _{v \rightarrow \infty} J_{v}(v, w, t) .
$$

We are interested in computing the firing rate in the limit $t \rightarrow \infty$. In this limit, the probability distribution becomes time independent. We thus set $\partial_{t} P$ to zero in equation 2.3, resulting in a continuity equation involving only $v$ and $w$. That equation cannot be solved exactly, but it can be solved as an expansion in the ratio of the synaptic to the membrane time constant (or its inverse). To facilitate this expansion, we introduced a new variable, $z \equiv k w / \sigma$, where $k \equiv\left(\tau_{s} / \tau_{m}\right)^{1 / 2}$. The advantage of using $z$ rather than $w$ is that its variance remains $\mathcal{O}(1)$ in both the large and small $k$ limits, a property not shared by $w$. With this change of variable and with $\partial_{t} P$ set to zero, equation 2.3 may be written as

$$
L P(v, z)=k \sigma z \partial_{v} P(v, z)+k^{2} \partial_{v}[f(v) P(v, z)]
$$

where

$$
L \cdot \equiv \frac{1}{2} \partial_{z}^{2} \cdot+\partial_{z}(z \cdot)
$$

In the next two sections, we solve this equation in the short and long correlation time limits.

\section{Short Correlation Time Limit}

To solve equation 2.6 in the short correlation time limit $(k \ll 1)$, we expand both the probability distribution and the firing rate in powers of $k$,

$$
\begin{aligned}
& P(v, z)=\sum_{i=0}^{\infty} k^{i} P_{i}(v, z) \\
& v=\sum_{i=0}^{\infty} k^{i} v_{i s},
\end{aligned}
$$

where the subscript $S$ on $v_{i s}$ is to remind us that we are working in the short correlation time limit. (Note that $P_{i}$ should also have a subscript $S$; we do 
not include it for clarity. It should be clear from the context whether we are referring to the short or long correlation time limit; the latter is discussed in section 4.) Since $P(v, z)$ is a probability distribution, it must integrate to one, independent of $k$. Consequently, we must have

$$
\begin{aligned}
& \int d v d z P_{0}(v, z)=1 \\
& \int d v d z P_{i}(v, z)=0, \quad \text { for } i \geq 1 .
\end{aligned}
$$

To derive equations for the $P_{i}$, we insert equation 3.1a into equation 2.6 and match powers of $k$. This results in a coupled set of equations:

$$
\begin{aligned}
& L P_{0}(v, z)=0 \\
& L P_{1}(v, z)=\sigma z \partial_{v} P_{0}(v, z) \\
& L P_{i}(v, z)=\sigma z \partial_{v} P_{i-1}(v, z)+\partial_{v}\left[f(v) P_{i-2}(v, z)\right], \quad \text { for } i \geq 2 .
\end{aligned}
$$

Using equations $2.4 \mathrm{a}, 2.5$, and $3.1 \mathrm{~b}$, the firing rate at order $i$ is

$$
\begin{aligned}
v_{i s} & =\frac{1}{\tau_{m}} \int_{-\infty}^{+\infty} d z \lim _{v \rightarrow \infty}\left[f(v) P_{i}(v, z)+\sigma z P_{i+1}(v, z)\right] \\
& =\frac{1}{\tau_{m}} \int_{-\infty}^{+\infty} d z \lim _{v \rightarrow \infty} f(v) P_{i}(v, z),
\end{aligned}
$$

where the last equality follows because $P(v, z)$ must vanish as $v \rightarrow \infty$; otherwise, it will not be normalizable.

3.1 Probability Distribution in the Short Correlation Time Limit. To compute the probability distribution as an expansion in powers of $k$, we simply solve equations 3.3a through 3.3c order by order. The zeroth-order solution, $P_{0}$, corresponds to white noise; the next orders give corrections associated with the finite synaptic time constant. It turns out, as we will see, that we have to go to the fourth order to determine the lowest nonvanishing correction to the firing rate. In this section, however, we sketch the solutions to equations 3.3a through 3.3c only through second order; the solutions through fourth order are derived in appendix A. We begin with equation 3.3a.

Examining equation 3.3a, we see that the zeroth-order contribution to the probability distribution, $P_{0}$, can be written as a linear combination of two functions: one even in $z$ and the other odd. Those two functions, denoted $\phi_{0}$ and $\phi_{1}$, are given by

$$
\begin{aligned}
& \phi_{0}(z)=\pi^{-1 / 2} \exp \left(-z^{2}\right) \\
& \phi_{1}(z)=\exp \left(-z^{2}\right) \int_{0}^{z} \exp \left(u^{2}\right) d u .
\end{aligned}
$$


The second of these, $\phi_{1}(z)$, scales as $1 / z$ for large $z$. Consequently, it will be negative when $z$ approaches either $+\infty$ or $-\infty$, and large compared to $\phi_{0}$. This violates the condition $P(v, z) \geq 0$ (recall that $P(v, z)$ is a probability distribution). Thus, $P_{0}$ must be proportional to $\phi_{0}$ only. The constant of proportionality can be $v$-dependent, so we have

$$
P_{0}(v, z)=Q_{0}(v) \phi_{0}(z) .
$$

The function $Q_{0}(v)$ is determined at higher order, as we will see shortly.

The first-order correction to the probability distribution, $P_{1}(v, z)$, is found by combining equations $3.3 \mathrm{~b}$ and 3.5 :

$$
P_{1}(v, z)=Q_{1}(v) \phi_{0}(z)+\sigma \partial_{v} Q_{0}(v) L^{-1} z \phi_{0}(z) .
$$

Because we explicitly included the homogeneous term in the above expression, $L^{-1}$ must pick out only inhomogeneous terms. This leads to

$$
L^{-1} F(z)=2 \exp \left(-z^{2}\right) \int_{0}^{z} d z^{\prime} \exp \left(z^{\prime 2}\right) \int_{-\infty}^{z^{\prime}} d z^{\prime \prime} F\left(z^{\prime \prime}\right),
$$

which is valid for any L1 function $F(z)$ that vanishes at $\pm \infty$. The lower limit of $-\infty$ in the second integral ensures that $L^{-1} F(z)$ does not pick up a piece proportional to $\phi_{1}(z)$, as we will see below.

The second-order correction, $P_{2}(v, z)$, which comes from combining equations 3.3c, 3.5, and 3.6, may be written, after a small amount of algebra, as

$$
\begin{aligned}
P_{2}(v, z)= & Q_{2}(v) \phi_{0}(z)+\sigma \partial_{v} Q_{1}(v) L^{-1} z \phi_{0}(z) \\
& +\sigma^{2} \partial_{v}^{2} Q_{0}(v) L^{-1} z L^{-1} z \phi_{0}(z)+\partial_{v}\left[f(v) Q_{0}(v)\right] L^{-1} \phi_{0}(z) .
\end{aligned}
$$

Using equation 3.7 , it is straightforward to show that

$$
\begin{aligned}
& L^{-1} z \phi_{0}(z)=-z \phi_{0}(z) \text { and } \\
& \left(L^{-1} z\right)^{2} \phi_{0}(z)=\left(z^{2} / 2\right) \phi_{0}(z)-(1 / 2) L^{-1} \phi_{0}(z) .
\end{aligned}
$$

With these relations, equation 3.8 becomes

$$
\begin{aligned}
P_{2}(v, z)= & Q_{2}(v) \phi_{0}(z)-\sigma \partial_{v} Q_{1}(v) z \phi_{0}(z)+\frac{\sigma^{2}}{2} \partial_{v}^{2} Q_{0}(v) z^{2} \phi_{0}(z) \\
& -\left[\frac{\sigma^{2}}{2} \partial_{v}^{2} Q_{0}(v)-\partial_{v}\left[f(v) Q_{0}(v)\right]\right] L^{-1} \phi_{0}(z) .
\end{aligned}
$$

Again using equation 3.7, we see that for large, positive $z, L^{-1} \phi_{0}(z) \sim 1 /|z|$. This makes $L^{-1} \phi_{0}(z)$ nonnormalizable, which means that it cannot make 
any contribution to $P_{2}(v, z)$. Thus, the solvability condition on equation 3.9 is

$$
\frac{\sigma^{2}}{2} \partial_{v}^{2} Q_{0}(v)-\partial_{v}\left[f(v) Q_{0}(v)\right]=0 .
$$

This solvability condition can also be derived by applying the Fredholm alternative theorem (Reed \& Simon, 1980).

To solve equation 3.10, we first integrate once with respect to $v$ and multiply both sides by -1 , yielding

$$
f(v) Q_{0}(v)-\frac{\sigma^{2}}{2} \partial_{v} Q_{0}(v)=\tau_{m} v_{0 s} .
$$

In this equation, we chose a specific form for the constant of integration (the term on the right-hand side). To see that this is the correct choice, note that in the large $v$ limit, $\partial_{v} Q_{0}(v)$ must approach zero to ensure that $Q_{0}$ is normalizable. Thus, in this limit, the only term that survives on the lefthand side is $f(v) Q_{0}(v)$. Multiplying both sides by $\tau_{m}^{-1} \phi_{0}(z)$, integrating over $z$, and taking the limit $v \rightarrow \infty$, equation 3.11 becomes equation 3.4 with $i=0$, indicating that $\tau_{m} v_{0 s}$ is the correct constant of integration.

Equation 3.11 has the solution

$$
Q_{0}(v)=\frac{2 v_{0 s} \tau_{m}}{\sigma^{2}} \int_{v}^{\infty} d u \exp [\psi(v)-\psi(u)]
$$

where

$$
\psi(v) \equiv \int_{0}^{v} \frac{2 f(u)}{\sigma^{2}} d u .
$$

The value of $v_{0 \mathrm{~s}}$ is determined from the condition that $Q_{0}(v)$ must be normalized to 1 (see equation 3.2a and note that $\int d z \phi_{0}(z)=1$ ), which implies that

$$
\nu_{0 \mathrm{~s}}=\frac{\sigma^{2}}{2 \tau_{m}}\left[\int_{-\infty}^{\infty} d v \int_{v}^{\infty} d u \exp [\psi(v)-\psi(u)]\right]^{-1} .
$$

We will compute this integral explicitly in the next section.

To determine the next order correction to the firing rate, we need to find $Q_{1}(v)$. This is done by deriving a solvability condition at third order. Similarly, $Q_{2}(v)$, which is needed for the second-order correction, is determined by deriving a solvability condition at fourth order. Deriving these conditions requires that we compute $P_{3}$ and $P_{4}$. This is conceptually no more difficult than computing $P_{1}$ and $P_{2}$; the only real difference is that the algebra is slightly more involved. We thus relegate those computations to 
appendix A. There, we find that $Q_{1}(v)=0$, which means that the first-order correction to the firing rate, $v_{1 s}$, vanishes, and that $Q_{2}(v)$ is given by

$$
\begin{aligned}
Q_{2}(v)= & \frac{v_{2 s}}{v_{0 \mathrm{~s}}} Q_{0}(v)-\frac{\tau_{m} \nu_{0 s}}{\sigma^{2}} \int_{v}^{\infty} d u \\
& \times \exp [\psi(v)-\psi(u)]\left[h(u)-h(v)+4 f^{\prime}(u)\right]
\end{aligned}
$$

where

$$
h^{\prime}(v) \equiv-3 f^{\prime \prime}(v)-4 f^{\prime}(v) \psi^{\prime}(v) .
$$

To calculate $v_{2 s}$, we use equation $3.2 \mathrm{~b}$, which tells us that $\int d v d z P_{2}(v, z)=0$, and equation 3.9, along with the facts that the coefficient in front of $L^{-1} \phi_{0}$ vanishes, $\int_{-\infty}^{\infty} d v \partial_{v}^{2} Q_{0}(v)=0$, and $\int_{-\infty}^{\infty} d v \partial_{v} Q_{0}(v)=0$, which tell us that the only way for $P_{2}(v, z)$ to integrate to zero is to have $\int_{-\infty}^{\infty} d v Q_{2}(v)=0$. Then, integrating both sides of equation 3.15 and using $\int_{-\infty}^{\infty} d v Q_{0}(v)=1$, we arrive at

$$
\begin{aligned}
v_{2 s}= & v_{0 s} \frac{\tau_{m} \nu_{0 s}}{\sigma^{2}} \int_{-\infty}^{\infty} d v \int_{v}^{\infty} d u \\
& \times \exp [\psi(v)-\psi(u)]\left[h(u)-h(v)+4 f^{\prime}(u)\right] .
\end{aligned}
$$

3.2 Firing Rate in the Short Correlation Time Limit. The zeroth-order contribution to the firing rate is given by equation 3.14 , and the lowest nonvanishing correction, which enters at $\mathcal{O}\left(k^{2}\right)$, is given by equation 3.17. These expressions involve double integrals and thus are not so easy to compute. However, we show in appendix B that both can be reduced to a single integral, resulting in

$$
\begin{aligned}
& \nu_{0 s}=\frac{1}{\pi \tau_{m}}\left[\int_{-\infty}^{\infty} \frac{d \xi}{\pi^{1 / 2}} \exp \left[-\mu \xi^{2}-\sigma^{4} \xi^{6} / 48\right]\right]^{-1} \\
& \nu_{2 s}=-v_{0 s} \pi \sigma^{2} \frac{\tau_{m} v_{0 s}}{2} \int_{-\infty}^{\infty} \frac{d \xi}{\pi^{1 / 2}} \xi^{2} \exp \left[-\mu \xi^{2}-\sigma^{4} \xi^{6} / 48\right] .
\end{aligned}
$$

The firing rate through second order, which we denote $v_{S}$ to indicate that the expression is valid in the short correlation time limit, is then given by

$$
v_{\mathrm{S}}=v_{0 \mathrm{~s}}+k^{2} v_{2 \mathrm{~s}} .
$$

In the absence of noise $(\sigma=0)$, the $\sqrt{\mu}$ behavior is recovered for the zeroth-order contribution to the firing rate,

$$
\nu_{0 \mathrm{~s}}=\frac{\mu^{1 / 2}}{\pi \tau_{m}},
$$




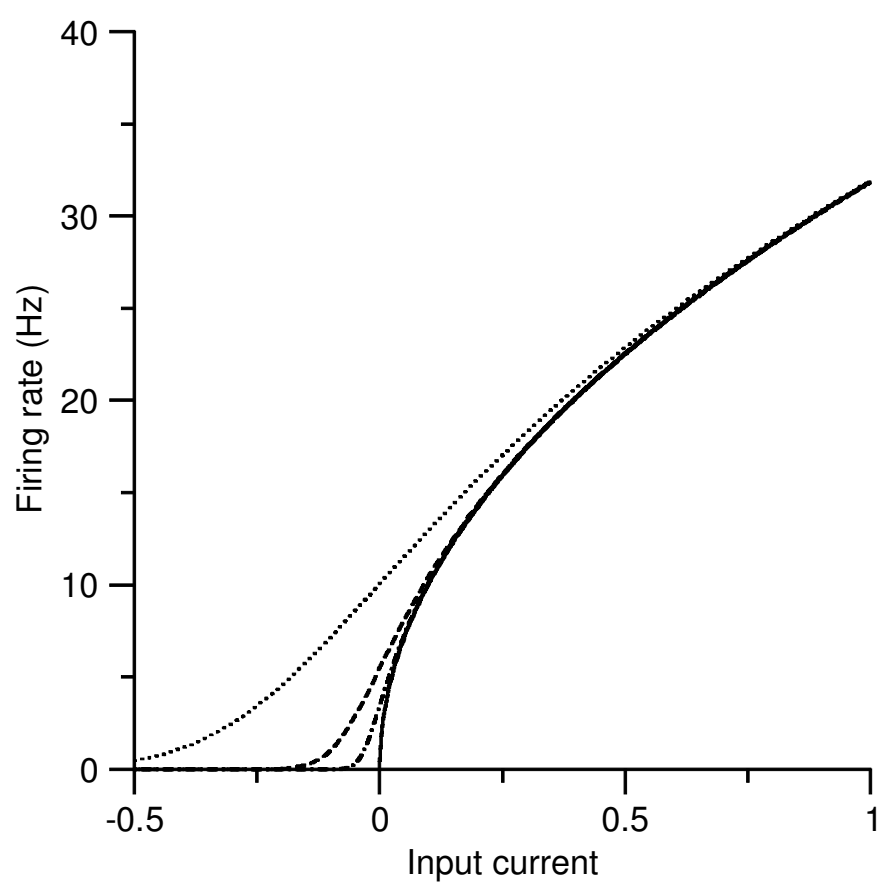

Figure 1: Firing rate as a function of mean input current, $\mu$, for white noise with $\sigma=0$ (solid line), 0.1 (dot-dashed line), 0.2 (dashed line), and 0.5 (dotted line). $\tau_{m}=10 \mathrm{~ms}$.

and in the absence of a mean current $(\mu=0), \nu_{0}$ increases with noise as $\sigma^{2 / 3}$,

$$
v_{0 s}=\frac{3^{5 / 6} \sigma^{2 / 3}}{2^{2 / 3} \pi^{1 / 2} \Gamma(1 / 6) \tau_{m}} .
$$

Plots of $\nu_{0 \mathrm{~s}}$ versus $\mu$ are given in Figure 1 for several values of $\sigma$.

The second-order contribution to the firing rate, $\nu_{2 s}$ (the lowest nonvanishing contribution), is negative. Thus, the firing rate decreases linearly with $k^{2}\left(=\tau_{s} / \tau_{m}\right)$ as the correlation time constant increases. This is inconvenient, as the total firing rate through second order, equation 3.19, can become negative at large $k$. It is possible to derive alternative expressions that coincide up to second order in $k$ with equation 3.19 but stay positive for any value of $k$. One such alternative expression is

$$
v_{\mathrm{s}}=\frac{v_{0 \mathrm{~s}}}{1-k^{2} v_{2 \mathrm{~s}} / \nu_{0 \mathrm{~s}}},
$$


which, after a small amount of algebra, reduces to

$$
v_{\mathrm{s}}=\frac{1}{\pi \tau_{m}}\left[\int_{-\infty}^{\infty} \frac{d \xi}{\pi^{1 / 2}} \exp \left[-\mu \xi^{2}-\sigma^{4} \xi^{6} / 48\right]\left(1+k^{2} \sigma^{2} \xi^{2} / 2\right)\right]^{-1} \text {. }
$$

A second alternative expression can be derived by replacing $1+k^{2} \sigma^{2} \xi^{2} / 2$ with $\exp \left(k^{2} \sigma^{2} \xi^{2} / 2\right)$ in equation 3.21, which leads to the expression

$$
\nu_{\mathrm{S}}=\frac{1}{\pi \tau_{m}}\left[\int_{-\infty}^{\infty} \frac{d \xi}{\pi^{1 / 2}} \exp \left[-\left(\mu-k^{2} \sigma^{2} / 2\right) \xi^{2}-\sigma^{4} z^{6} / 48\right]\right]^{-1} .
$$

Both equations 3.20 and 3.22 have the same behavior at small $k$ as equation 3.19. However, they have the advantage over equation 3.19 that the firing rate stays positive for any value of $\tau_{s}$.

Equations 3.20 and 3.22 are plotted in Figure 2, along with the results of numerical simulations of equation 2.1. Numerical simulations were done using the theta model (Gutkin \& Ermentrout, 1998), which is related to the quadratic neuron by the change of variables $v=\tan (\theta / 2)$. In the theta model, spikes are identified by passage of $\theta$ through $\pi$. The equations were integrated using a Euler scheme with $d t=0.001 \tau_{m}$. Note that the fact that noise is colored avoids the complications associated with numerical integration of the multiplicative white noise, where Ito or Stratonovich prescriptions yield different results (Lindner, Longtin, \& Bulsara, 2003).

Figure 2 shows the firing rate as a function of the ratio $\tau_{s} / \tau_{m}$ for three different regimes: suprathreshold $(\mu>0)$, threshold $(\mu=0)$, and subthreshold $(\mu<0)$. In the suprathreshold regime, the second-order expansion holds only for small $\tau_{s}$ (roughly speaking, $\tau_{s}<0.5 \tau_{m}$ ), and when $\tau_{s}$ becomes of order $\tau_{m}$ or larger, both equations 3.20 and 3.22 underestimate the firing rate. When $\mu=0$, equation 3.20 gives a very good approximation to the firing rate in a large range of $\tau_{s}$, at least up to $\tau_{s}=2 \tau_{m}$. Equation 3.22, on the other hand, strongly underestimates the firing rate unless $\tau_{s} / \tau_{m}$ is small. Finally, when $\mu$ is sufficiently subthreshold, equation 3.22 gives a good approximation to the firing rate, while equation 3.20 overestimates it.

Although both equations 3.20 and 3.22 have advantages, in the remainder of this article, we focus on the former. This is because it more easily merges with the long correlation time firing rate (which we derive in the next section), and can thus be used to provide an expression for the firing rate that is approximately valid for all synaptic time constants.

\section{The Long Correlation Time Limit}

In the suprathreshold regime, the short synaptic time expansion describes the firing rate only when $\tau_{s}$ is small compared to $\tau_{m}$, that is, only when $k$ is small. This does not mean we cannot compute the firing rate when $k$ is 

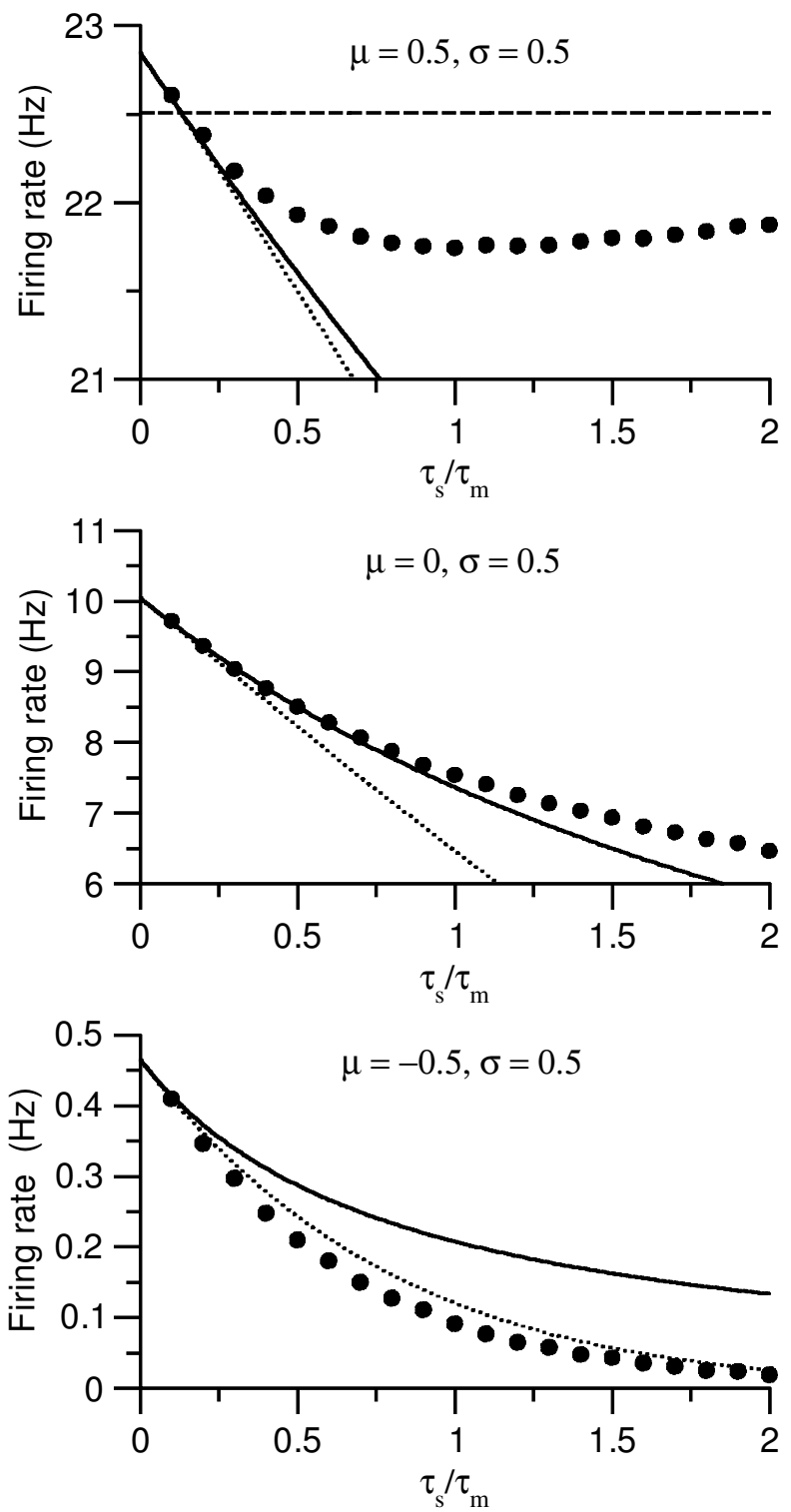

Figure 2: Firing rate as a function of synaptic time constant for three different input statistics (indicated at the top of each graph). In each graph, solid curve: equation 3.20; dotted curve: equation 3.22; full circles: simulations (error bars are smaller than symbol size in all cases); dashed horizontal curve in the upper panel: firing rate for $\sigma=0$ or $\tau_{s}=\infty . \tau_{m}=10 \mathrm{~ms}$. 
large; it just means that we need to perform an expansion in powers of $1 / k$ rather than $k$. Analogous to equations 3.1a and 3.1b, we write

$$
\begin{aligned}
& P(v, z)=\sum_{i=0}^{\infty} k^{-i} P_{i}(v, z) \\
& v=\sum_{i=0}^{\infty} k^{-i} v_{i \mathrm{~L}},
\end{aligned}
$$

where the subscript $L$ is to remind us that we are working in the long correlation limit. As mentioned in section 3, the $P_{i}$ in equation 4.1a are different from the ones in equation 3.1a, but it should be clear what we mean from the context. To ensure that $P(v, z)$ has the proper normalization, we demand that the $P_{i}$ obey the conditions given in equation 3.2.

To derive the equations for the $P_{i}$, we insert equation 4.1a into equation 2.6 and match powers of $k$. We find that the $P_{i}$ obey the relations

$$
\begin{aligned}
& \partial_{v} f(v) P_{0}(v, z)=0 \\
& \partial_{v} f(v) P_{1}(v, z)=-\sigma z \partial_{v} P_{0} \\
& \partial_{v} f(v) P_{i}(v, z)=-\sigma z \partial_{v} P_{i-1}+L P_{i-2}, \quad \text { for } i \geq 2 .
\end{aligned}
$$

In the next section, we solve these equations through second order, and in the section after that, we compute the firing rate, again through second order (the lowest nonvanishing order).

4.1 Probability Distribution in the Long Correlation Time Limit. The strategy for solving equations $4.2 \mathrm{a}$ through $4.2 \mathrm{c}$ is essentially identical to that used for equations 3.3a through 3.3c: compute $P_{i}$ at each order by inverting a differential operator, express $P_{i}$ in terms of a set of arbitrary functions, and determine those functions by deriving solvability conditions at higher order. The analysis is, however, considerably easier in the long than the short correlation time limit, for two reasons. First, the operator $\partial_{v}[f(v) \cdot]$, which appears on the left-hand side of equations 4.2 a through $4.2 c$, is much easier to invert than $L$, the corresponding operator for equations 3.3a through 3.3c. Second, we need solve for $P_{i}$ only through third order rather than fourth.

We begin by writing down the solutions of equations 4 .2a through $4.2 \mathrm{c}$ through second order, which are easily found by inspection:

$$
\begin{aligned}
& P_{0}(v, z)=\frac{p_{0}(z)}{f(v)} \\
& P_{1}(v, z)=\frac{p_{1}(z)}{f(v)}-\frac{\sigma z p_{0}(z)}{f^{2}(v)} \\
& P_{2}(v, z)=\frac{p_{2}(z)}{f(v)}-\frac{\sigma z p_{1}(z)}{f^{2}(v)}+\frac{\sigma^{2} z^{2} p_{0}(z)}{f^{3}(v)}+\frac{L p_{0}(z)}{f(v)} \int^{v} \frac{d u}{f(u)} .
\end{aligned}
$$


The $p_{i}(z)$ are constants of integration that arise from inverting the operator $\partial_{v}[f(v) \cdot]$; they are analogous to the $Q_{i}(v)$ that appeared in the short correlation time analysis.

Note that equations 4.3a through $4.3 \mathrm{c}$ are valid only for $\mu>0$. For $\mu<0$, the distribution at zero order is a delta function at $v=-\sqrt{-\mu}$, and the firing rate is $v_{0 L}=0$. Thus, in the following, we consider the case $\mu>0$; we will come back to the subthreshold regime in section 5 .

From equations $4.3 \mathrm{a}$ and $4.3 \mathrm{~b}$, we see that the probability flux, $f(v) P_{i}(v, z)$, is continuous at $z= \pm \infty$ when $i=0$ or 1 . However, this is not the case for equation $4.3 \mathrm{c}$, for which there is a jump discontinuity in the second-order flux,

$$
\left.f(v) P_{2}(v, z)\right|_{v=+\infty}-\left.f(v) P_{2}(v, z)\right|_{v=-\infty}=L p_{0}(z) \int_{-\infty}^{\infty} \frac{d u}{f(u)} .
$$

To eliminate the discontinuity, we must have $L p_{0}(z)=0$. Using the same reasoning that led to equation 3.5 , this implies that

$$
p_{0}(z)=\frac{\mu^{1 / 2}}{\pi} \phi_{0}(z)
$$

The factor $\mu^{1 / 2} / \pi$ was chosen to ensure that $\int d v d z P_{0}(v, z)=1$, which follows from $\int d z \phi_{0}(z)=1$ and $\int d v / f(v)=\pi \mu^{-1 / 2}$. With $\operatorname{Lp}_{0}(z)=0, P_{2}(v, z)$ is given by

$$
P_{2}(v, z)=\frac{p_{2}(z)}{f(v)}-\frac{\sigma z p_{1}(z)}{f^{2}(v)}+\frac{\sigma^{2} z^{2} p_{0}(z)}{f^{3}(v)} .
$$

With the last term in equation $4.3 \mathrm{c}$ eliminated, we find, by combining equations $4.2 \mathrm{c}, 4.3 \mathrm{~b}$, and 4.5 , that $P_{3}(v, z)$ is given by

$$
\begin{aligned}
P_{3}(v, z)= & \sum_{j=0}^{3} \frac{(-\sigma z)^{j} p_{3-j}(z)}{f^{j+1}(v)} \\
& +\frac{\operatorname{Lp}(z)}{f(v)} \int^{v} \frac{d u}{f(u)}-\frac{\sigma L z p_{0}(z)}{f(v)} \int^{v} \frac{d u}{f^{2}(u)} .
\end{aligned}
$$

Again, there is a jump discontinuity in the probability flux:

$$
\begin{aligned}
f(v) & \left.P_{3}(v, z)\right|_{v=+\infty}-\left.f(v) P_{3}(v, z)\right|_{v=-\infty}=\operatorname{Lp} p_{1}(z) \int_{-\infty}^{\infty} \frac{d u}{f(u)} \\
& -\sigma L z p_{0}(z) \int_{-\infty}^{\infty} \frac{d u}{f^{2}(u)} .
\end{aligned}
$$


To determine how to choose $p_{1}(z)$ to eliminate the discontinuity, we use

$$
\begin{aligned}
\int_{-\infty}^{\infty} \frac{d v}{f^{n}(v)} & =\frac{\left(-\partial_{\mu}\right)^{n-1}}{(n-1) !} \int_{-\infty}^{\infty} \frac{d v}{f(v)}=\frac{\pi}{(n-1) !}\left(-\partial_{\mu}\right)^{n-1} \frac{1}{\sqrt{\mu}} \\
& =\frac{\pi}{\mu^{1 / 2}} \frac{(2 n-3) ! !}{(2 \mu)^{n-1}(n-1) !},
\end{aligned}
$$

where the first and second equalities follow from the fact that $f(v)=v^{2}+\mu$ (see equation 2.2), and $n ! !$ is defined implicitly via $n ! ! \equiv n \times(n-2) ! !$ and $(-1) ! ! \equiv 1$. Then, using equation 4.8 to compute the integrals over $u$ in equation 4.7, we find that the discontinuity in the third-order flux vanishes when $p_{1}(z)$ obeys the equation $L p_{1}(z)=(\sigma / 2 \mu) L z p_{0}(z)$. Consequently,

$$
p_{1}(z)=\frac{\sigma}{2 \mu}\left[z p_{0}(z)+c_{1} \phi_{0}(z)\right]
$$

where $c_{1}$ is an arbitrary constant. To determine the constant, we use the fact that

$$
\int d v d z P_{1}(v, z)=0
$$

which tells us that $c_{1}=0$.

Knowledge of $p_{1}(z)$ is, it turns out, sufficient to calculate the lowest nonvanishing correction to the firing rate. We turn now to that calculation.

4.2 Firing Rate in the Long Correlation Time Limit. In the long correlation time limit, the probability distribution at each order can be written as an expansion in powers of $1 / f(v)$. In fact, it is not hard to show that

$$
P_{i}(v, z)=\sum_{j=0}^{i} \frac{(-\sigma z)^{j} p_{i-j}(z)}{f^{j+1}(v)},
$$

which is a straightforward generalization of equation 4.6 with all terms that produce jump discontinuities at $\pm \infty$ removed. Thus, since $f(v) \sim v^{2}$ for large $v$ (see equation 2.2), the $i$ th order probability flux at $v=+\infty$ is proportional to $p_{i}(z)$. This in turn means, via equation 3.4, that the $i$ th order firing rate is given by

$$
v_{i \mathrm{~L}}=\frac{1}{\tau_{m}} \int_{-\infty}^{\infty} d z p_{i}(z) .
$$

Thus, to compute the firing rate at order $i$, we need to know only the integral of the $p_{i}$, which simplifies the analysis somewhat. 
To compute $v_{0_{\mathrm{L}}}$, we use equation 4.10 with $i=0$ and equation 4.4 for $p_{0}(z)$, and we find that

$$
\nu_{0 \mathrm{~L}}=\frac{\mu^{1 / 2}}{\pi \tau_{m}}
$$

As stated above, $v_{0 \mathrm{~L}}=0$ when $\mu<0$, which means that the long correlation time limit affects only the suprathreshold regime. To compute $v_{1}$, we use equation 4.10 with $i=1$ and equation 4.9 for $p_{1}(z)$. Performing the integral in equation 4.10, we find that $v_{1 \mathrm{~L}}=0$.

With $v_{1}=0$, we need to go to second order to find a correction to $v_{0 \mathrm{~L}}$. At this order, the situation changes slightly, since we do not have an explicit expression for $p_{2}(z)$. However, we can find the integral of $p_{2}(z)$, which is all we need for $v_{2 \mathrm{~L}}$, by integrating both sides of equation 4.5. Applying equation $3.2 \mathrm{~b}$, which tells us that this integral is zero, and using equation 4.8 for the integrals over $v$, we arrive at

$$
\int d z p_{2}(z)=\frac{\sigma}{2 \mu} \int d z z p_{1}(z)-\frac{3 \sigma^{2}}{8 \mu^{2}} \int d z z^{2} p_{0}(z)
$$

With equations 4.4 and 4.9 for $p_{0}(z)$ and $p_{1}(z)$, the integrals are elementary, and we find, via equation 4.10 , that

$$
\nu_{2 \mathrm{~L}}=-\frac{\mu^{1 / 2}}{\pi \tau_{m}} \frac{\sigma^{2}}{16 \mu^{2}}
$$

We will adopt the convention that $\nu_{2 \mathrm{~L}}=\infty$ if $\mu \leq 0$.

Thus, the firing rate through second order in $1 / k$ is given by

$$
v_{\mathrm{L}}=\frac{\mu^{1 / 2}}{\pi \tau_{m}}\left(1-\frac{\sigma^{2}}{16 \mu^{2} k^{2}}\right) .
$$

Interestingly, in the long correlation time limit, noise decreases the firing rate. This is the opposite of what we saw in the short correlation time limit, where, even at zeroth order, noise increases the firing rate (see equation 3.18a).

Equation 4.11 suffers from the same inconvenience as equation 3.19: it becomes negative for small $k$. An alternative expression that is identical to it through second order in $1 / k$ is

$$
v_{\mathrm{L}}=\frac{\mu^{1 / 2}}{\pi \tau_{m}} \frac{1}{1+\sigma^{2} /\left(16 \mu^{2} k^{2}\right)} .
$$

Figure 3 shows how equation 4.12 compares with simulations. 

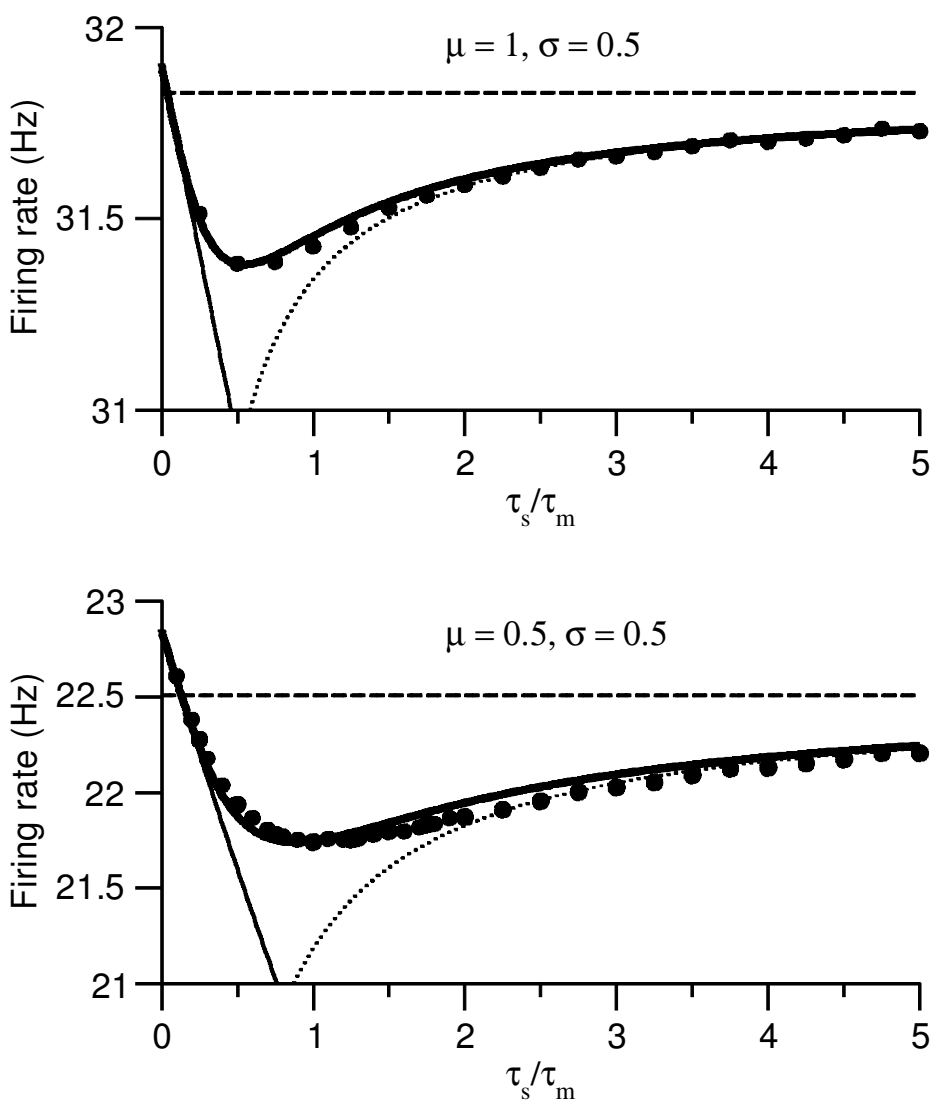

Figure 3: Firing rate as a function of synaptic time constant in the suprathreshold regime. Filled circles: simulations (error bars are smaller than symbol size in all cases). Thin solid lines: short synaptic time expansion (equation 3.20). Dotted line: long synaptic time expansion (equation 4.12). Long-dashed line: firing rate for $\sigma=0$ or $\tau_{s}=\infty$. Thick solid line: simple rational function that interpolates between short and long synaptic time constants, equation 5.1.

\section{Combining the Short and Long Correlation Time Limits}

One might wonder if a simple function could interpolate between the short and the long synaptic time expansions. The simplest function that reproduces the calculated asymptotics is a rational function of the form

$$
v(k)=\frac{\nu_{0 \mathrm{~s}}+a k^{2}+b \nu_{0 \mathrm{~L}} k^{4}}{1+c k^{2}+b k^{4}} .
$$


This function was chosen so that it asymptotes to $v_{0 \mathrm{~s}}$ when $k \rightarrow 0$ and $v_{0 \mathrm{~L}}$ when $k \rightarrow \infty$. The three parameters, $a, b$, and $c$, are chosen by requiring that

$$
\begin{array}{ll}
v(k) \sim_{k \rightarrow 0} & v_{0 \mathrm{~s}}+v_{2 \mathrm{~s}} k^{2} \\
v(k) \sim_{k \rightarrow \infty} & v_{0 \mathrm{~L}}+v_{2 \mathrm{~L}} k^{-2} .
\end{array}
$$

Since there are only two conditions and three parameters, we have an additional degree of freedom when choosing $a, b$, and $c$. We can break the degeneracy associated with this degree of freedom by demanding that in the subthreshold regime, where $v_{0 \mathrm{~L}}=0, v(k)$ reduce exactly to equation 3.20. This forces $a$ to be zero, and we find that

$$
v(k)=\frac{v_{0 \mathrm{~s}}+\left(v_{2 \mathrm{~s}} / \nu_{0 \mathrm{~s}}\right)\left(v_{0 \mathrm{~L}} / \nu_{2 \mathrm{~L}}\right) v_{0 \mathrm{~L}} k^{4}}{1-\left(v_{2 \mathrm{~s}} / \nu_{0 \mathrm{~s}}\right) k^{2}+\left(v_{2 \mathrm{~s}} / \nu_{0 \mathrm{~s}}\right)\left(v_{0 \mathrm{~L}} / \nu_{2 \mathrm{~L}}\right) k^{4}} .
$$

Because both $\nu_{2 \mathrm{~S}}$ and $\nu_{2 \mathrm{~L}}$ are negative and $\nu_{0 \mathrm{~S}}$ and $\nu_{0 \mathrm{~L}}$ are positive, $v(k)$ is never negative.

Figure 3 shows that equation 5.2 provides a good approximation to the simulated data in the suprathreshold regime, even in the intermediate range (around $\tau_{s}=\tau_{m}$ ) where both short and long time expansions fail. In the subthreshold regime, $v_{0_{\mathrm{L}}} / \nu_{2_{\mathrm{L}}}$ is zero, and equation 5.2 reduces to equation 3.20. Thus, we can evaluate how well $v(k)$ approximates the true firing rate in the subthreshold regime by examining the solid line in Figure 2. Although $v(k)$ slightly overestimates the firing rate, the overestimation is small in absolute terms, mainly because the firing rate is small.

Interestingly, the synaptic time constant has different effects on the firing rate in the subthreshold and suprathreshold regimes. In the subthreshold regime (see Figure 2), the firing rate decreases monotonically with synaptic time constant. In the suprathreshold range (see Figure 3), however, the dependence is nonmonotonic: firing rate decreases with time constant for small $\tau_{s}$ but increases with large $\tau_{s}$, and in between there is a minimum.

The overall quality of the interpolation can be seen from the $f$-I curve, which is plotted in Figure 4 for $\tau_{s} / \tau_{m}=0.5$ and $\tau_{s} / \tau_{m}=2$. This plot indicates that equation 5.2 does a good approximation of the true firing rate at all values of $\mu$, both subthreshold and suprathreshold.

As can be seen from Figure 4, colored and white noise produce about the same firing rate in the suprathreshold regime. This is not surprising, since noise has very little effect in the suprathreshold regime, whatever its characteristics (all curves approach the dotted line when the input current is large). In the subthreshold regime, however, the firing rate with colored noise is small compared to that with white noise. For example, when $\tau_{s}=20$ $\mathrm{ms}$ and $\tau_{m}=10 \mathrm{~ms}$ (both reasonable values for real neurons), the ratio of the colored noise firing rate to the white noise firing rate is 0.37 when the input current is -0.25 . 


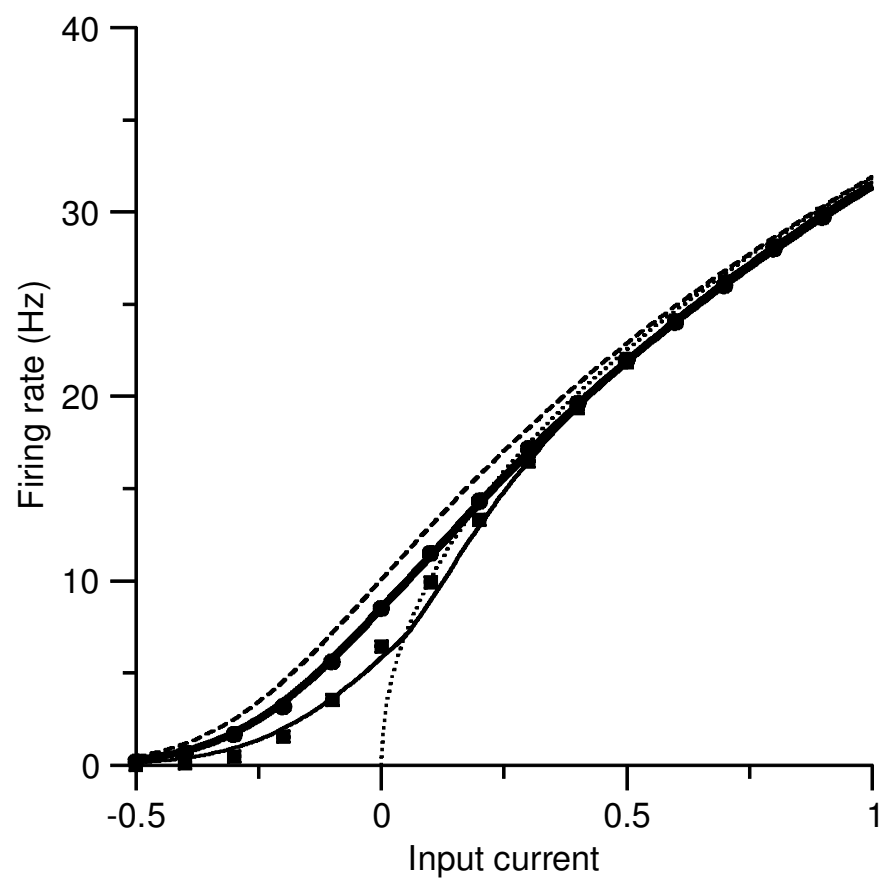

Figure 4: Firing rate as a function of mean input current, $\mu$, when $\sigma=0.5$, $\tau_{m}=10 \mathrm{~ms}$, and at several values of the synaptic time constant. Curves are from equation 5.1, symbols are simulations. Dashed line: $\tau_{s}=0$ (white noise). Circles/thick solid curve: $\tau_{s}=5 \mathrm{~ms}$ (colored noise). Squares/thin solid curve: $\tau_{s}=20 \mathrm{~ms}$ (colored noise). Dotted line: $\tau_{s}=\infty$ (no noise). Error bars for the simulations are smaller than the symbol size in all cases.

A second difference between colored and white noise is the dependence of the firing rate on the amplitude of the noise, $\sigma$. For short correlation times, $\tau_{s} \ll \tau_{m}$, increasing the noise amplitude increases the firing rate (see equation 3.18a, from which it is easy to see that $v_{0 \mathrm{~s}}$ is an increasing function of $\sigma$ ). For long correlation times, on the other hand, noise decreases firing rate (see equation 4.12). This difference is important for models that propose noise as a mechanism of gain control (Chance, Abbott, \& Reyes, 2002; Prescott \& De Koninck, 2003).

To compute firing rate via equation 5.2, there are two quantities that must be determined numerically: $v_{0 s}$ and $v_{2 s}$, the zeroth- and second-order firing rates in the short correlation time limit. Fortunately, both of these quantities depend primarily on a single variable, $\gamma=48^{1 / 3} \mu / \sigma^{4 / 3}$. Using equations 
3.18a and $3.18 \mathrm{~b}$, it is straightforward to show that

$$
\begin{aligned}
& \nu_{0 s}=\frac{1}{\pi \tau_{m}}\left(\frac{\sigma^{4}}{48}\right)^{1 / 6} \frac{1}{\psi_{0}(\gamma)} \\
& \nu_{2 s}=-\pi \sqrt{12} \tau_{m} v_{0 s}^{2} \psi_{2}(\gamma),
\end{aligned}
$$

where

$$
\psi_{p}(\gamma) \equiv \int_{-\infty}^{\infty} \frac{d \xi}{\pi^{1 / 2}} \xi^{p} \exp \left[-\gamma \xi^{2}-\xi^{6}\right] .
$$

Thus, the firing rate of the quadratic integrate-and-fire neuron in the presence of colored noise depends on two functions, $\psi_{0}(\gamma)$ and $\psi_{2}(\gamma)$. This is extremely convenient for applications in which rapid calculation of firing rates is important (such as mean-field calculations): $\psi_{0}(\gamma)$ and $\psi_{2}(\gamma)$ can be computed for a range of values of $\gamma$ and put into lookup tables, and the firing rates for all values of $\tau_{s}, \tau_{m}, \mu$, and $\sigma$ can then be rapidly computed using those tables.

\section{Discussion}

The firing rate of the noisy quadratic integrate-and-fire (QIF) neuron was computed using expansions in the ratio of the noise correlation time to the neuronal time constant, $\tau_{s} / \tau_{m}$. Our approach was to first write down the Fokker-Planck equation for the time evolution of the voltage and synaptic current, the two variables describing the neuron. We then expanded this equation in either $\tau_{s} / \tau_{m}$ or $\tau_{m} / \tau_{s}$. In both limits, small $\tau_{s} / \tau_{m}$ and small $\tau_{m} / \tau_{s}$, the Fokker-Planck equation reduced to an infinite set of ordinary differential equations (ODEs). These ODEs could be solved at successive orders by recursion.

The calculation for short correlation times turned out to be much simpler for the quadratic than for the more standard linear integrate-and-fire (LIF) neuron (Brunel \& Sergi, 1998; Fourcaud \& Brunel, 2002). This is because the main difficulty with the LIF is that the voltage is reset from threshold to zero in a rather unnatural way, which leads to complicated boundary conditions for the Fokker-Planck equation. With the QIF, the boundary conditions are much more natural $(+\infty$ is identified with $-\infty)$, which dramatically simplifies the calculation.

Because of the different boundary conditions, the firing rate of the QIF neuron is much less sensitive to temporal correlations in the noise than the LIF neuron. Indeed, at small $\tau_{s}$, the firing rate for the QIF neuron decreases as $\tau_{s} / \tau_{m}$ rather than $\sqrt{\tau_{s} / \tau_{m}}$, as it does for the LIF neuron. At large correlation times and large input currents, on the other hand, the behavior of the QIF and LIF neurons is qualitatively similar (Moreno, de la Rocha, Renart, \& Parga, 2002; Moreno \& Parga, 2003). 
The problem we studied is a particular case of the calculation of the mean first passage time of a dynamical system passing through a saddlepoint bifurcation. This problem has been studied extensively over the past several decades. Sigeti and Horsthemke (1989), Colet, San Miguel, Casademunt, and Sancho (1989), and Lindner, Longtin, and Bulsara (2003) studied the problem for white noise, and derived an expression analogous to our equation 3.14. Ramírez-Piscina \& Sancho (1991), using other methods, considered the weakly colored noise case and derived our equations 3.18a and 3.18b. These results have been extended here in three ways: we have presented an alternative computation of the mean first passage time in the short correlation time limit, we have obtained the mean first passage time in the long correlation time limit, and we showed how a simple analytical formula can interpolate between the two limits and provide a good approximation of the firing rates for any correlation time.

These results extend our knowledge of the basic properties of the QIF model. This model has seen increasing interest in recent years among theoreticians, primarily because the QIF neuron has the same properties as any type I neuron whenever the firing rate is low (Ermentrout \& Kopell, 1986; Ermentrout, 1996). Among other studies, Roper, Bressloff, and Longtin (2000) studied the critical slowing down and stochastic resonance/trapping effects that occur close to the saddle-node bifurcation. QIF neurons have also been used in network studies: Latham, Richmond, Nelson, and Nirenberg (2000) studied the equilibrium properties of the background state of large networks, and Hansel and Mato $(2001,2003)$ studied the synchronization properties of networks of QIF neurons in the absence of noise. In this context, our results can be useful in at least two ways. First, they can be used in the mean-field analysis of large networks of QIF neurons in the presence of noise, as has been done with LIF neurons (Amit \& Brunel, 1997a, 1997b; Brunel, 2000). Second, they can be used to understand the trade-off between noise and synaptic and membrane time constants in type I neurons. This is important, as synaptic noise may be a mechanism for changing the gain of neurons, and may even act as a form of gain control (Chance et al., 2002; Prescott \& De Koninck, 2003).

\section{Appendix A: Correction to the Probability Distribution Through Fourth Order in $k$ in the Short Correlation Time Limit}

In this appendix, we compute $P_{3}(v, z)$ and $P_{4}(v, z)$, which allows us to compute the correction to the firing rate through second order (the lowest nonvanishing correction). To simplify the calculation, we define the two linear operators,

$$
\begin{aligned}
& L_{1} \equiv \sigma L^{-1} z \partial_{v} \\
& L_{2} \equiv L^{-1} \partial_{v} f(v)
\end{aligned}
$$


where $L^{-1}$ is given by equation 3.7 and both $L^{-1}$ and $\partial_{v}$ operate on everything to their right-for example, $L_{2} g(v, z)=L^{-1} \partial_{v}[f(v) g(v, z)]$. With this notation, the solution to equation $3.3 \mathrm{c}$ is

$$
P_{i}(v, z)=Q_{i}(v) \phi_{0}(z)+L_{1} P_{i-1}(v, z)+L_{2} P_{i-2}(v, z) .
$$

Iterating equation A. 2 several times with $i=3$, it is straightforward to show that

$$
P_{3}=Q_{3} \phi_{0}+L_{1} Q_{2} \phi_{0}+\left(L_{1}^{2}+L_{2}\right) Q_{1} \phi_{0}+\left[L_{1}\left(L_{1}^{2}+L_{2}\right)+L_{2} L_{1}\right] Q_{0} \phi_{0} .
$$

For clarity, we have suppressed the arguments; our convention is that $Q_{i}$ is a function of $v$ and $\phi_{0}$ is a function of $z$.

In what follows we will need to compute $L^{-1} z^{n} \phi_{0}$ through $n=4$; these quantities are given, via equation 3.7, by

$$
\begin{aligned}
L^{-1} z \phi_{0}(z) & =-z \phi_{0}(z) \\
L^{-1} z^{2} \phi_{0}(z) & =-\left(z^{2} / 2\right) \phi_{0}(z)+(1 / 2) L^{-1} \phi_{0}(z) \\
L^{-1} z^{3} \phi_{0}(z) & =-\left(z^{3} / 3+z\right) \phi_{0}(z) \\
L^{-1} z^{4} \phi_{0}(z) & =-\left(z^{4} / 4+3 z^{2} / 4\right) \phi_{0}(z)+(3 / 4) L^{-1} \phi_{0}(z) .
\end{aligned}
$$

Using equations A.1 and A.3a through A.3c it is straightforward to show that

$$
\begin{aligned}
P_{3}= & {\left[Q_{3}-\sigma z \partial_{v} Q_{2}(v)-\frac{\sigma^{3}}{2}\left(\frac{z^{3}}{3}+z\right) \partial_{v}^{3} Q_{0}(v)+\sigma z \partial_{v}\left[f(v) \partial_{v} Q_{0}(v)\right]\right] } \\
& \times \phi_{0}(z)+\frac{\sigma^{2}}{2} \partial_{v}^{2} Q_{1} z^{2} \phi_{0}-\left[\frac{\sigma^{2}}{2} \partial_{v}^{2} Q_{1}-\partial_{v} f Q_{1}\right] L^{-1} \phi_{0} .
\end{aligned}
$$

Since the coefficient in front of $L^{-1} \phi_{0}$ must vanish, $Q_{1}(v)$ obeys the same differential equation as $Q_{0}(v)$ (see equation 3.10). However, $Q_{1}(v)$ differs from $Q_{0}(v)$ in that the former integrates to zero while the latter integrates to 1 (see equations 3.2a and 3.2b). This can happen only if $Q_{1}$ vanishes. In fact, it is not hard to show in general that $Q_{i}$ vanishes for $i$ odd. We will not prove this here, but we will assume it in the remainder of this appendix. Thus, both $Q_{1}(v)$ and $Q_{3}(v)$ are zero, and $P_{3}$ is given by

$$
P_{3}=\left[-\sigma z \partial_{v} Q_{2}(v)-\frac{\sigma^{3}}{2}\left(\frac{z^{3}}{3}+z\right) \partial_{v}^{3} Q_{0}(v)+\sigma z \partial_{v}\left[f(v) \partial_{v} Q_{0}(v)\right]\right] \phi_{0}(z) .
$$

We now turn to $P_{4}$. Iterating equation A.2 with both $Q_{1}$ and $Q_{3}$ zero, we arrive at

$$
P_{4}=Q_{4} \phi_{0}+\left(L_{1}^{2}+L_{2}\right) Q_{2} \phi_{0}+\left[\left(L_{1}^{2}+L_{2}\right)^{2}+L_{1} L_{2} L_{1}\right] Q_{0} \phi_{0} .
$$


Using equation A. 1 for $L_{1}$ and $L_{2}$ and equation A.3 to invert $L$, we find after straightforward, but tedious, algebra that

$$
\begin{aligned}
P_{4}= & Q_{4} \phi_{0}+\partial_{v} f Q_{2} z^{2} \phi_{0}+\frac{\sigma^{4}}{24} \partial_{v}^{4} Q_{0} z^{4} \phi_{0} \\
& +\left[\frac{\sigma^{2}}{2} \partial_{v}^{2} Q_{2}-\partial_{v} f Q_{2}+\frac{3 \sigma^{4}}{8} \partial_{v}^{4} Q_{0}-\frac{\sigma^{2}}{2} \partial_{v}^{2} f \partial_{v} Q_{0}-\frac{\sigma^{2}}{4} \partial_{v} f \partial_{v}^{2} Q_{0}\right] \\
& \times\left(z^{2} \phi_{0}-L^{-1} \phi_{0}\right) .
\end{aligned}
$$

The solvability condition on $Q_{2}$ is thus

$$
\frac{\sigma^{2}}{2} \partial_{v}^{2} Q_{2}-\partial_{v} f Q_{2}=-\frac{3 \sigma^{4}}{8} \partial_{v}^{4} Q_{0}+\frac{\sigma^{2}}{2} \partial_{v}^{2} f \partial_{v} Q_{0}+\frac{\sigma^{2}}{4} \partial_{v} f \partial_{v}^{2} Q_{0}
$$

Inserting equation A.5 into A.4, we arrive at

$$
P_{4}=Q_{4} \phi_{0}+\partial_{v} f Q_{2} z^{2} \phi_{0}+\frac{\sigma^{4}}{24} \partial_{v}^{4} Q_{0} z^{4} \phi_{0} .
$$

To find $Q_{2}(v)$, we first simplify the right-hand side of equation A.5. Using equations 3.10 and 3.12, equation A.5 may be rewritten

$$
\frac{\sigma^{2}}{2} \partial_{v}^{2} Q_{2}-\partial_{v} f Q_{2}=-\frac{\sigma^{2}}{4} \partial_{v}\left[3 f^{\prime \prime} Q_{0}+4 f^{\prime} \psi^{\prime} Q_{0}-\frac{8 v_{0} \tau_{m}}{\sigma^{2}} f^{\prime}\right],
$$

where a prime denotes a derivative.

To solve this equation, we use the same method as with equation 3.10: we integrate both sides with respect to $v$ and multiply by -1 . This produces

$$
\begin{aligned}
f(v) Q_{2}(v)-\frac{\sigma^{2}}{2} \partial_{v} Q_{2}(v) \\
=\tau_{m} \nu_{2 s}-\left[\frac{\sigma^{2}}{4}\left[-3 f^{\prime \prime}(v)-4 f^{\prime}(v) \psi^{\prime}(v)\right] Q_{0}(v)+2 v_{0 s} \tau_{m} f^{\prime}\right] .
\end{aligned}
$$

The constant of integration was set to $\tau_{m} \nu_{2 s}$. To see why, note first of all that we can combine equations 3.4 and 3.9 to write

$$
\tau_{m} v_{2 s}=\lim _{v \rightarrow \infty}\left[f(v) Q_{2}(v)+\frac{\sigma^{2}}{4} f(v) \partial_{v}^{2} Q_{0}(v)\right]=\lim _{v \rightarrow \infty} f(v) Q_{2}(v),
$$

where the first equality follows from the facts that $Q_{1}(v)=0$, the coefficient in front of $L^{-1} \phi_{0}$ vanishes, and $\int_{-\infty}^{\infty} z^{2} \phi_{0}(z)=1 / 2$. The second equality follows because in the large $v$ limit, $Q_{0} \sim 1 / f$ and $f \sim v^{2}$. Consequently, equation A.6 is correct if both $\partial_{v} Q_{2}$ and the term in brackets on the right-hand 
side of that equation vanish as $v \rightarrow \infty$. The first of these, $\partial_{v} Q_{2}$, must vanish at large $v$ to ensure normalizability. To verify that the term in brackets vanishes, use $\lim _{v \rightarrow \infty} Q_{0}(v)=v_{0 s} \tau_{m} / f(v)$ (see equation 3.11 and the discussion following it) to write

$$
\begin{gathered}
\lim _{v \rightarrow \infty}\left[\frac{\sigma^{2}}{4}\left[-3 f^{\prime \prime}(v)-4 f^{\prime}(v) \psi^{\prime}(v)\right] Q_{0}(v)+2 v_{0 s} \tau_{m} f^{\prime}\right] \\
=v_{0 s} \tau_{m} \lim _{v \rightarrow \infty}\left[-\frac{3 \sigma^{2} f^{\prime \prime}}{4 f}-\frac{\sigma^{2} f^{\prime} \psi^{\prime}}{f}+2 f^{\prime}\right] .
\end{gathered}
$$

Using equation 3.13 , which tells us that $\psi^{\prime}=2 f / \sigma^{2}$, the last two terms on the right-hand side cancel. This leaves us with $f^{\prime \prime} / f$, which vanishes for large $v$. Thus, equation A.6 in the large $v$ limit really is equivalent to equation A.7, and $\nu_{2 s}$ is the correct constant of integration.

Equation A.6 has the solution

$$
\begin{aligned}
Q_{2}(v)= & \frac{v_{2 \mathrm{~s}}}{v_{0 \mathrm{~s}}} Q_{0}(v)-\int_{v}^{\infty} d u \exp [\psi(v)-\psi(u)] \\
& \times\left[\frac{1}{2} h^{\prime}(u) Q_{0}(u)+\frac{4 v_{0 s} \tau_{m}}{\sigma^{2}} f^{\prime}(u)\right],
\end{aligned}
$$

where $h(v)$ is given in equation 3.21. This can be simplified by integrating the first term in brackets by parts and using equation 3.12 for $Q_{0}(v)$; the resulting expression is given in equation 3.15.

\section{Appendix B: Simplifying the Expressions for Firing Rate in the Short Correlation Time Limit}

The expressions for the zeroth- and second-order firing rates in the short correlation time limit, equations 3.14 and 3.17, consist of rather complicated two-dimensional integrals. We can transform them into one-dimensional integrals by making the change of variables,

$$
\begin{aligned}
& v=x-y \\
& u=x+y,
\end{aligned}
$$

and integrating over $x$.

With this change of variables, for which the Jacobian is 2, equation 3.14 becomes

$$
\nu_{0 s}=\frac{\sigma^{2}}{4 \tau_{m}}\left[\int_{-\infty}^{\infty} d x \int_{0}^{\infty} d y \exp [\psi(x-y)-\psi(x+y)]\right]^{-1} .
$$


For the quadratic integrate-and-fire neuron, $f(v)=v^{2}+\mu$, equation 2.2, and we have, via equation 3.13 ,

$$
\psi(v)=\frac{2}{\sigma^{2}}\left[\frac{v^{3}}{3}+\mu v\right] .
$$

Inserting equation B. 3 into B.2 and performing the integral over $x$, we find that

$$
\nu_{0 \mathrm{~s}}=\frac{\sigma}{2 \pi \tau_{m}}\left[\int_{0}^{\infty} \frac{d y}{\pi^{1 / 2}} y^{-1 / 2} \exp \left[-4 \mu y / \sigma^{2}-4 y^{3} / 3 \sigma^{2}\right]\right]^{-1} .
$$

Finally, letting $y=\sigma^{2} \xi^{2} / 4$, we arrive at equation 3.18a.

We now turn to the expression for the second-order contribution to the firing rate, $v_{2 s}$, equation 3.17. This equation is more difficult to reduce to a one-dimensional integral because of the term $h(u)-h(v)+f 4^{\prime}(u)$ in the integrand. Our first step, then, is to simplify this term. Integrating equation 3.16, we find that $h(v)=-2 f(v) \psi^{\prime}(v)-3 f^{\prime}(v)$, so that

$$
h(u)-h(v)+f 4^{\prime}(u)=-2\left[f(u) \psi^{\prime}(u)-f(v) \psi^{\prime}(v)\right]+f^{\prime}(u)+3 f^{\prime}(v) .
$$

The first term on the right-hand side can be further modified by integrating equation 3.17 by parts,

$$
\begin{gathered}
\int_{-\infty}^{\infty} d v \int_{v}^{\infty} d u \exp [\psi(v)-\psi(u)]\left[f(u) \psi^{\prime}(u)-f(v) \psi^{\prime}(v)\right] \\
=\int_{-\infty}^{\infty} d v\left[\int_{v}^{\infty} d u \exp [\psi(v)-\psi(u)] f(u) \psi^{\prime}(u)\right. \\
\left.\quad-\int_{-\infty}^{v} d u \exp [\psi(u)-\psi(v)] f(u) \psi^{\prime}(u)\right] \\
=\int_{-\infty}^{\infty} d v \int_{v}^{\infty} d u \exp [\psi(v)-\psi(u)]\left[f^{\prime}(u)+f^{\prime}(v)\right] .
\end{gathered}
$$

The intermediate step is useful because it allows explicit cancellation of infinities that appear in the naive integration by parts. Using equation B.5, we can replace equation B. 4 with $-f^{\prime}(u)+f^{\prime}(v)$, and equation 3.17 becomes

$$
v_{2 s}=-v_{0 s} \frac{\tau_{m} v_{0 s}}{\sigma^{2}} \int_{-\infty}^{\infty} d v \int_{v}^{\infty} d u \exp [\psi(v)-\psi(u)]\left[f^{\prime}(u)-f^{\prime}(v)\right]
$$

Again making the change of variables given in equation B.1, using $f(v)=$ $v^{2}+\mu$, and integrating over $x$, equation $B .6$ becomes

$$
\nu_{2 s}=-4 v_{0 s} \frac{\tau_{m} \nu_{0 s}}{\sigma^{2}}\left(\pi \sigma^{2}\right)^{1 / 2} \int_{0}^{\infty} d y y^{1 / 2} \exp \left[-4 \mu y / \sigma^{2}-4 y^{3} / 3 \sigma^{2}\right] .
$$

Finally, letting $y=\sigma^{2} \xi^{2} / 4$, we arrive at equation $3.18 \mathrm{~b}$. 


\section{Acknowledgments}

We acknowledge the hospitality of the Institute for Theoretical Physics at the University of California, Santa Barbara, where this work was performed. N.B. thanks Ruben Moreno for discussing his results on the long synaptic time expansion of the leaky integrate-and-fire neuron. P.L. was supported by NIMH grant R01 MH62447.

\section{References}

Abbott, L. F., \& van Vreeswijk, C. (1993). Asynchronous states in a network of pulse-coupled oscillators. Phys. Rev. E, 48, 1483-1490.

Amit, D. J., \& Brunel, N. (1997a). Dynamics of a recurrent network of spiking neurons before \& following learning. Network, 8, 373-404.

Amit, D. J., \& Brunel, N. (1997b). Model of global spontaneous activity and local structured activity during delay periods in the cerebral cortex. Cerebral Cortex, 7, 237-252.

Amit, D. J., \& Tsodyks, M. V. (1991). Quantitative study of attractor neural network retrieving at low spike rates I: Substrate—spikes, rates and neuronal gain. Network, 2, 259-274.

Anderson, J. S., Lampl, I., Gillespie, D. C., \& Ferster, D. (2000). The contribution of noise to contrast invariance of orientation tuning in cat visual cortex. Science, 290, 1968-1972.

Brunel, N. (2000). Dynamics of sparsely connected networks of excitatory \& inhibitory spiking neurons. J. Comput. Neurosci., 8, 183-208.

Brunel, N., \& Hakim, V. (1999). Fast global oscillations in networks of integrate\&-fire neurons with low firing rates. Neural Computation, 11, 1621-1671.

Brunel, N., \& Sergi, S. (1998). Firing frequency of integrate-\&-fire neurons with finite synaptic time constants. J. Theor. Biol., 195, 87-95.

Burns, B. D., \& Webb, A. C. (1976). The spontaneous activity of neurones in the cat's cerebral cortex. Proc. R. Soc. Lond. B, 194, 211-223.

Chance, F., Abbott, L., \& Reyes, A. (2002). Gain modulation from background synaptic input. Neuron, 35, 773-782.

Colet, P., San Miguel, M., Casademunt, J., \& Sancho, J. M. (1989). Relaxation from a marginal state in optical bistability. Phys. Rev. A, 39, 149-156.

Destexhe, A., \& Paré, D. (1999). Impact of network activity on the integrative properties of neocortical pyramidal neurons in vivo. J. Neurophysiol., 81, 1531-1547.

Destexhe, A., Rudolph, M., Fellous, J.-M., \& Sejnowski, T. J. (2001). Fluctuating synaptic conductances recreate in vivo-like activity in neocortical neurons. Neuroscience, 107, 13-24.

Ermentrout, G. B. (1996). Type I membranes, phase resetting curves, \& synchrony. Neural Computation, 8, 979-1001.

Ermentrout, G. B., \& Kopell, N. (1986). Parabolic bursting in an excitable system coupled with a slow oscillation. SIAM J. Appl. Math., 46, 233-253.

Fourcaud, N., \& Brunel, N. (2002). Dynamics of firing probability of noisy integrate-\&-fire neurons. Neural Computation, 14, 2057-2110. 
Gutkin, B., \& Ermentrout, G. B. (1998). Dynamics of membrane excitability determine interspike interval variability: A link between spike generation mechanisms and cortical spike train statistics. Neural Comp., 10, 1047-1065.

Hansel, D., \& Mato, G. (2001). Existence and stability of persistent states in large neuronal networks. Phys. Rev. Lett., 10, 4175-4178.

Hansel, D., \& Mato, G. (2003). Asynchronous states and the emergence of synchrony in large networks of interacting excitatory and inhibitory neurons. Neural Comp., 15, 1-56.

Knight, B. W., Omurtag, A., \& Sirovich, L. (2000). The approach of a neuron population firing rate to a new equilibrium: An exact theoretical result. Neural Computation, 12, 1045-1055.

Latham, P. E., Richmond, B. J., Nelson, P. G., \& Nirenberg, S. (2000). Intrinsic dynamics in neuronal networks. I. Theory. J. Neurophysiol., 83, 808-827.

Lindner, B., Longtin, A., \& Bulsara, A. (2003). Analytic expressions for rate and CV of a type I neuron driven by white gaussian noise. Neural Comp., in press.

Moreno, R., de la Rocha, J., Renart, A., \& Parga, N. (2002). Response of spiking neurons to correlated inputs. Phys. Rev. Lett., 89, 288101.

Moreno, R., \& Parga, N. (2003). Simple model neurons with slow filtering operate as detectors of rare events. Submitted.

Nykamp, D. Q., \& Tranchina, D. (2000). A population density approach that facilitates large-scale modeling of neural networks: Analysis and an application to orientation tuning. J. Comp. Neurosci., 8, 19-50.

Prescott, S., \& De Koninck, Y. (2003). Gain control of firing rate by shunting inhibition: Roles of synaptic noise and dendritic saturation. Proc. Natl. Acad. Sci., 100, 2076-2081.

Ramírez-Piscina, L., \& Sancho, J. M. (1991). First-passage times for a marginal state driven by colored noise. Phys. Rev. A, 43, 663-668.

Reed, M., \& Simon, S. (1980). Methods of modern mathematical physics I: Functional analysis. New York: Academic Press.

Ricciardi, L. M. (1977). Diffusion processes and related topics on biology. Berlin: Springer-Verlag.

Risken, H. (1984). The Fokker-Planck equation: Methods of solution and applications. Berlin: Springer-Verlag.

Roper, P., Bressloff, P. C., \& Longtin, A. (2000). A phase model of temperaturedependent mammalian cold receptors. Neural Comp., 12, 1067-1093.

Sigeti, D., \& Horsthemke, W. (1989). Pseudo-regular oscillations induced by external noise. J. Stat. Phys., 54, 1217.

Softky, W. R., \& Koch, C. (1993). The highly irregular firing of cortical cells is inconsistent with temporal integration of random EPSPs. J. Neurosci., 13, 334-350.

Treves, A. (1993). Mean-field analysis of neuronal spike dynamics. Network, 4, 259-284.

Tuckwell, H. C. (1988). Introduction to theoretical neurobiology. Cambridge: Cambridge University Press.

Received December 13, 2002; accepted April 4, 2003. 